ERRATA

\title{
QUANTUM MECHANICAL TREATMENT OF THE ELECTRICAL INTERACTION VIEWED AS AN ELASTIC COUPLING THROUGH FLUX LINES
}

\author{
[Int. J. Mod. Phys. B. Vol. 16, No. 32 (2002), 4809-4830] \\ ANDREI DOLOCAN and VOICU DOLOCAN
}

Pages 4809, 4820 and 4827:

"Linear crystal" should be "crystal".

Page 4812:

The right hand sides of Eqs. (10) and 911) should have a fractor of $\hbar N^{-12}$.

Page 4813:

The righthand sider of Eqs. (12) and (14) should hae a factor of $N^{-1}$.

In all formulae of the paper, $\eta$ should be replaced by $\hbar$. 